\title{
A comprehensive fluorescent sensor for spatiotemporal cell cycle analysis in Arabidopsis
}

\author{
Bénédicte Desvoyes $₫ \bowtie$, Ainhoa Arana-Echarri $\odot$, María D. Barea and Crisanto Gutierrez $₫$
}

\begin{abstract}
Assessing cell proliferation dynamics is crucial to understand the spatiotemporal control of organogenesis. Here we have generated a versatile fluorescent sensor, $\mathrm{PlaCCl}$ (plant cell cycle indicator) on the basis of the expression of CDT1a-CFP, H3.1-mCherry and CYCB1;1-YFP, that identifies cell cycle phases in Arabidopsis thaliana. This tool works in a variety of organs, and all markers and the antibiotic resistance are expressed from a single cassette, facilitating the selection in mutant backgrounds. We also show the robustness of $\mathrm{PlaCCl}$ line in live-imaging experiments to follow and quantify cell cycle phase progression.
\end{abstract}

Development in multicellular organisms depends on the highly regulated formation of new organs. Organogenesis requires the production of a sufficient number of cells in a temporally and spatially regulated manner, that eventually will constitute the cohort of different cell types present in the organ. Therefore, understanding morphogenesis requires the availability of confident markers to assess cell proliferation and identify unequivocally the different cell cycle phases in a living cell environment.

Efforts in several animal models have led to the development of the FUCCI (fluorescent, ubiquitination-based cell cycle indicator) systems that identify each cell cycle phase ${ }^{1-4}$. Comparable advances for live-imaging of cell proliferation studies in plants lag behind. One reason is that plants lack the CDT1a-interacting protein, geminin, a key component of the FUCCI system, although they contain a CDT1a-interacting protein (GEM) that is structurally unrelated to animal geminin ${ }^{5,6}$. Attempts to circumvent this have been reported. Thus, in addition to the tags used for $\mathrm{G} 2+\mathrm{M}$ (refs. ${ }^{7,8}$ ), the expression of a truncated version of CDT1a, a DNA replication initiator protein, from a heterologous promoter has been reported as an $\mathrm{S}+\mathrm{G} 2$ marker$^{9}$. In another approach, the fluorescent protein Venus fused to the destruction box of CYCB1;1 was expressed under the control of $\mathrm{H} 4$ promoter and labelled cells in S + G2 + M (ref. ${ }^{10}$ ). However, a comprehensive cell cycle marker line should ideally (1) be able to distinguish in an unequivocal manner all cell cycle phases to follow individual cells in a growing organ and (2) offer the possibility of using advanced live-imaging strategies. This is precluded when using nucleoside analogues for labelling the S-phase, since they require specific detection protocols incompatible with live imaging.

Still, one major drawback of cell cycle marker lines developed so far is the lack of appropriate markers to identify unequivocally cells in G1. We have recently analysed in detail CDT1a dynamics in proliferating cells and demonstrated that CDT1a starts to be loaded into chromatin shortly after mitosis, reaches a maximum during G1 and is rapidly degraded only minutes after S-phase initiation ${ }^{11}$, expanding previous claims of its proteasome-dependent degradation $^{12}$. Importantly, we demonstrated that CDT1a accumulates rapidly after the S-phase of endocycling cells and is detectable during the whole G-phase of the endocycle ${ }^{11}$. It was also shown that histone
H3.1 proteins, for example HTR13, expressed under their own promoters, are incorporated into chromatin from early S-phase $\mathrm{e}^{13}$ and that the dynamics of H3.1 in the root meristem differs depending on the proliferation potential of the cells. H3.1 is maintained during mitosis and the following G1 in cells with a high proliferation potential and exchanged in G2 in cells undergoing their last cell cycle $^{13}$. Thus, we reasoned that the use of the CDT1a, H3.1 and CYCB1;1-tagged lines would allow identification of G1, S + early $\mathrm{G} 2$ and late $\mathrm{G} 2+\mathrm{M}$ (prophase and metaphase) cells, respectively.

As a first proof of concept, we generated by crossing Arabidopsis plants expressing under their own promoters the three fluorescently labelled cell cycle proteins: CDT1a-CFP (ref. ${ }^{11}$ ), H3.1-mRFP (ref. ${ }^{13}$ ) and CYCB1;1-GFP (ref. ${ }^{14}$ ) (Supplementary Fig. 1a). Visualization by confocal microscopy of homozygous plants for the three markers clearly revealed nuclei labelled with the different fluorescent tags, for example, in the root apical meristem (Supplementary Fig. 1b): (1) nuclei in G1 with various intensities of CDT1a-CFP, (2) nuclei containing a H3.1-mRFP signal, revealing cells in S- or G2-phases, (3) a fraction among these also contained a CYCB1;1-GFP signal, indicative of late G2, (4) nuclei in prophase or metaphase also contained CYCB1;1-GFP (Supplementary Fig. 1c,d). Therefore, the simultaneous identification of all cell cycle phases provides a full picture of cell proliferation dynamics in the organ under study.

In this reporter plant, each marker is expressed from a different transgene and these, together with the antibiotic resistance gene, are inserted at different loci in the Arabidopsis genome. These facts limit its usefulness to analyse cell cycle regulation when multiple crosses and segregations steps are necessary, for example in mutant backgrounds. We solved this by using synthetic biology tools to generate a single construct carrying the three reporters ( $p$ CDT1a::CDT1a-eCFP, pHTR13::HTR13-mCherry and pCYCB1;1::NCYCB1;1-YFP) and only one antibiotic resistance gene ( $p N O S:: N P T I I)$. To this end we have used the Goldenbraid 3.0 strategy $^{15}$ to clone and assemble the different transcriptional units in a single vector (Supplementary Fig. 2, Supplementary Table 1 and Fig. 1a), which simplifies enormously the selection step. We confirmed that several independent lines expressing the three fluorescent markers performed similarly. We named this line PlaCCI, for plant cell cycle indicator, an acronym reminiscent of the FUCCI reporter developed for animal cells. We have already shown that the H3.1-GFP (ref. ${ }^{13}$ ) and CDT1a (ref. ${ }^{11}$ ) dynamics in endoreplicating cells are similar to that of proliferating cells.

We carried out live-imaging experiments to follow in detail cell cycle dynamics in the root meristem (Supplementary Video 1). Figure 1b shows an example of a cell in late G2 starting to express CYCB1;1-YFP with a H3.1-mCherry background (nucleus 1). After mitosis the two daughter cells started to accumulate CDT1a-CFP during G1, and finally CDT1a-CFP is degraded soon after initiating S-phase. In this case, the G1 length is $\sim 5-5.30 \mathrm{~h}$. Another cell in 
Multigene assembly cassette (Goldenbraid)

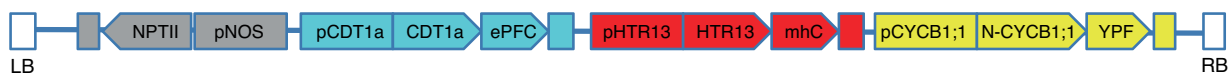

b
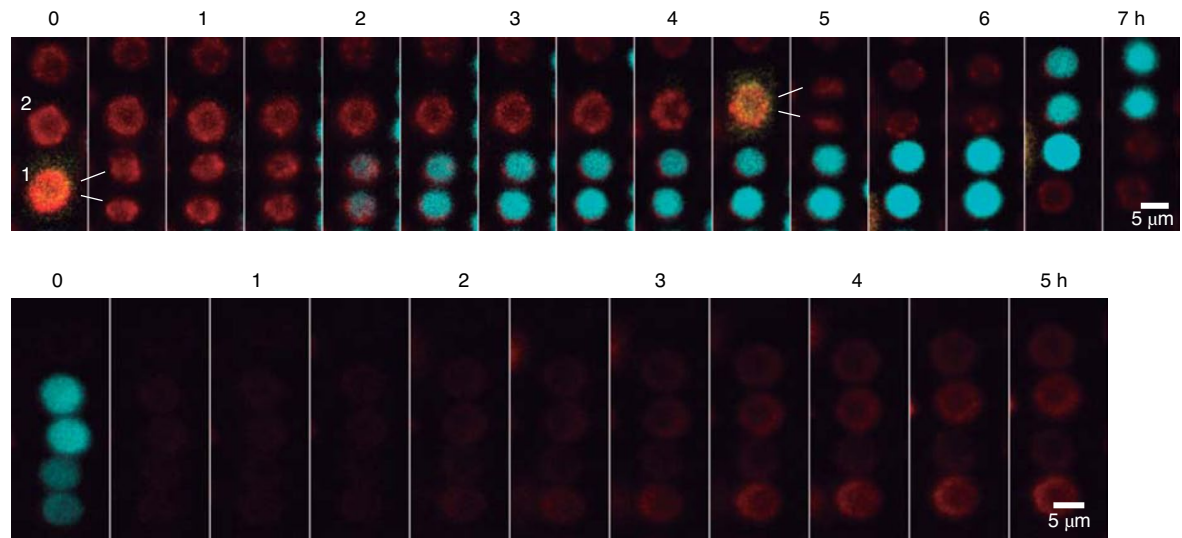

d

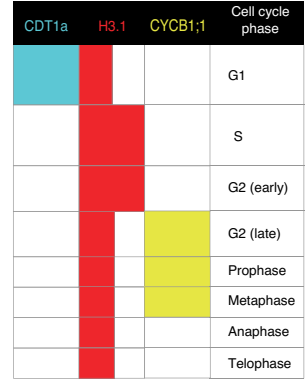

e
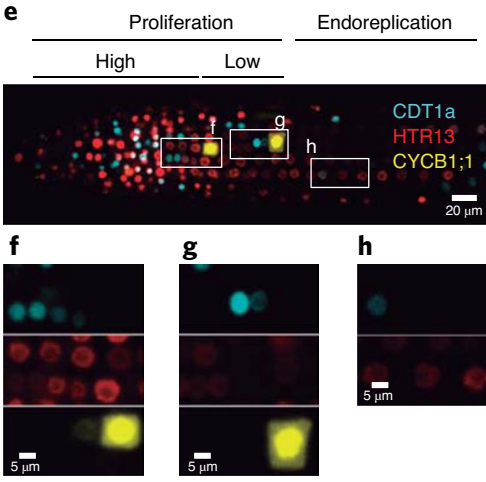

h
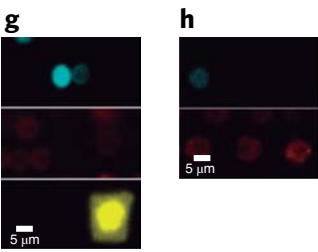

CDT1a

CYCB $1 ; 1$

- 20

i

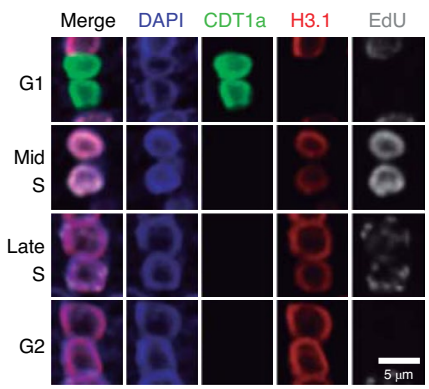

j

k

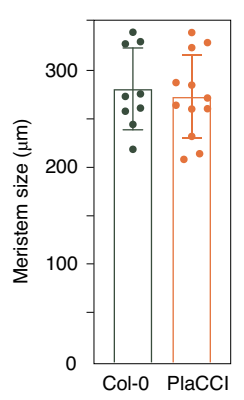

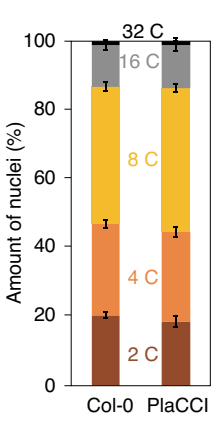

$I_{20}$

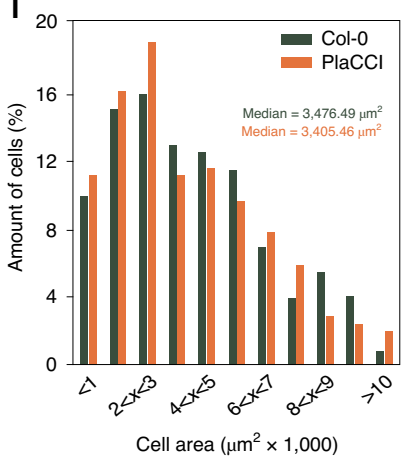

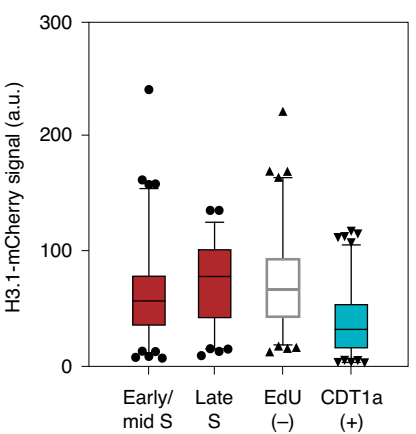

Fig. 1 | Development of PlaCCI. a, Goldenbraid multigene construct containing transcriptional units encoding kanamycin resistance gene, pCDT1a::CDT1a-eCFP, pHTR13:: pHTR13-mCherry and pCYCB1;1::NCYCB1;1-YFP, used to transform Arabidopsis Col-0 plants. RB, right border; LB, left border. b,c, Tracking of cell cycle stages in the root apical meristem using live imaging: two cells in late G2 (nucleus 1) and early G2 (nucleus 2), labelled in yellow and red at time 0 , divide and accumulate CDT1a-CFP in the following G1 (b); cells in G1, labelled with CDT1a-CFP, enter S-phase and accumulate HTR13-mCherry (c). Scale bars in b and c, $5 \mu \mathrm{m}$. d, The colour key used to identify cell cycle phases. Note that depending on the proliferative state of the cell, the G1 phase may or may not contain an H3.1-mCherry signal, as explained in the text. e-h, Confocal image of the root meristem of a plant expressing the three PlaCCl markers. In e, white boxes indicate the regions enlarged in $\mathbf{f}-\mathbf{h}$. Scale bar in $\mathbf{e}, 20 \mu \mathrm{m}$. $\mathbf{f}-\mathbf{h}$, Insets showing individual channels of parts of the root apical meristem located in the high proliferation rate (f), low proliferation rate where the last cell cycle takes place $(\mathbf{g})$ and endoreplication $(\mathbf{h})$ domains. Scale bars in $\mathbf{f}-\mathbf{h}, 5 \mu \mathrm{m}$. i, Upper panel, example of nuclei illustrating that EdU-pulse labelled nuclei contain H3.1-mCherry and that EdU and CDT1a-CFP signals do not colocalize (scale bar, $5 \mu \mathrm{m}$ ); bottom panel, distribution of H3.1-mCherry signal in EdU-labelled nuclei with patterns of early/mid $(n=99)$ and late $(n=79)$ S-phase, and for EdU-negative nuclei $(n=91)$ and CDT1a-CFP positive nuclei $(n=110)$. Boxes indicate the first and third quartiles and whiskers the maximum and minimum values of $95 \%$ of all nuclei. Dots represent the $5 \%$ of outliers. Lines in the boxes indicate the median values 58.05, 79.41, 67.83 and 33.31 for early/mid and late S-phase, EdU-negative and CDT1a-CFP positive nuclei, respectively. j, Root meristem size of 5-day-old Col-0 $(n=9)$ and $\mathrm{PlaCCl}(n=12)$ plants. Mean \pm s.d. are represented. Differences were not statistically significant $(P=0.671,95 \%$ confidence interval), using an unpaired $t$-test with Welch's correction. $\mathbf{k}$, Nuclear DNA ploidy profiles of leaves no. 3 and no. 4 of 28 -day-old Col-O $(n=4)$ and $\mathrm{PlaCCl}(n=6)$ plants. Mean \pm s.d. are represented. Differences between pairs of ploidy levels were not statistically different (multiple unpaired $t$-test $P$-values of $0.068,0.663,0.065,0.644,0.865$ for the $2 C, 4 C, 8 C, 16 C$ and $32 C$ values, respectively; $95 \%$ confidence interval). I, Cell area distribution of adaxial leaf epidermal cells of 28 -day-old Col-0 ( $n=199$ cells) and PlaCCI $(n=205$ cells) plants. The distributions were not different according to a $\chi^{2}$-test with $95 \%$ confidence. $\mathbf{b}, \mathbf{c}, \mathbf{e}-\mathbf{I}$, Experiments were repeated at least three times with similar results. 


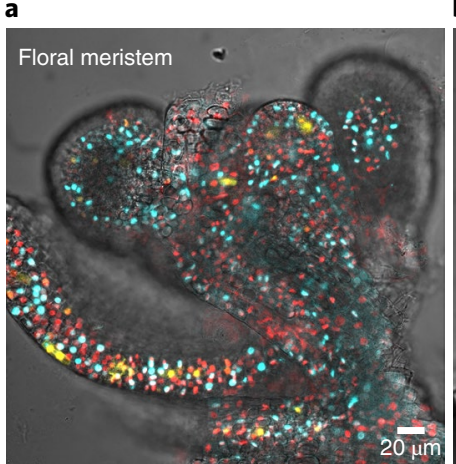

b
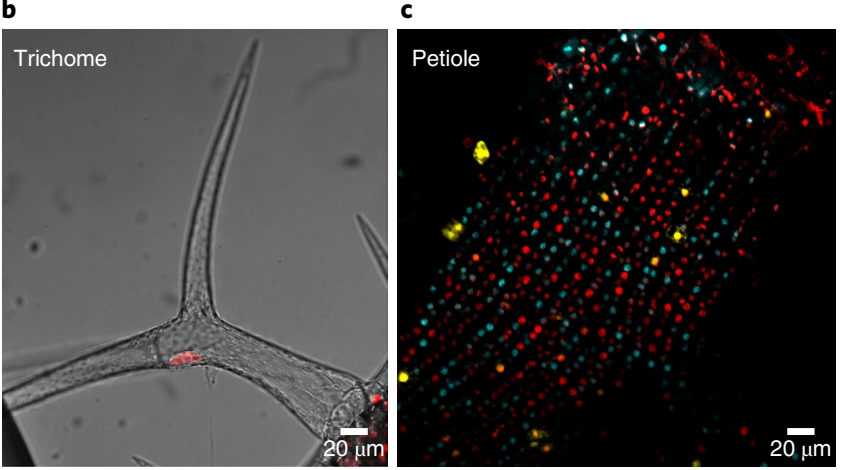

d

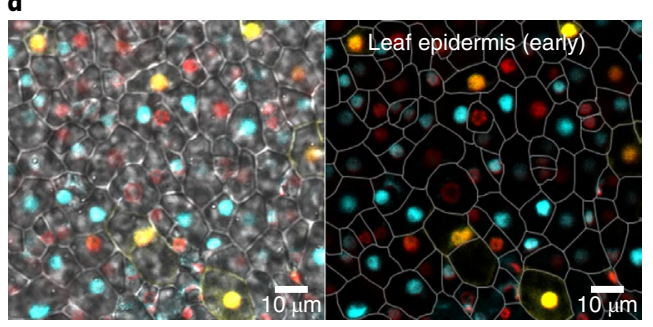

e
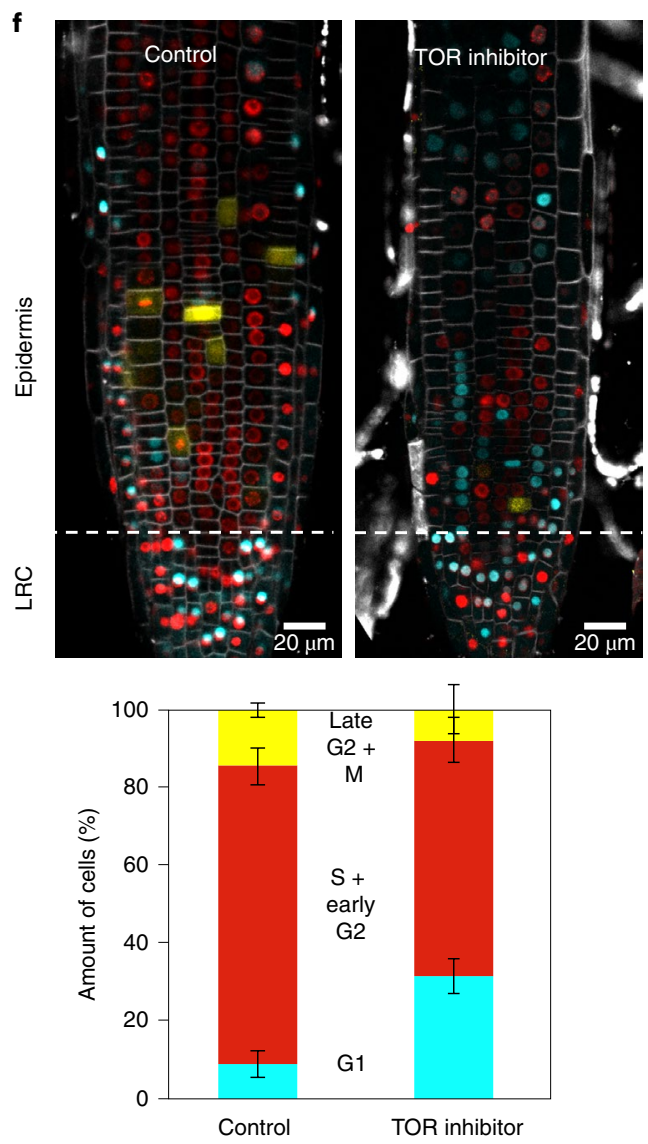

early G2 (nucleus 2), with a maximum amount of H3.1-mCherry, started accumulation of CYCB1;1-YFP, divided and initiated loading of CDT1a-CFP (Fig. 1b). Cells in G1, labelled with CDT1a-CFP, entered S-phase and accumulated H3.1-mCherry (Fig. 1c).

Quantifying each fluorescent signal separately in live-imaging experiments allowed us to identify clearly when the CDT1a-CFP and CYCB1;1-YFP markers are switched on/off (Supplementary Fig. 3). The overlaps of these signals with that of H3.1-mCherry

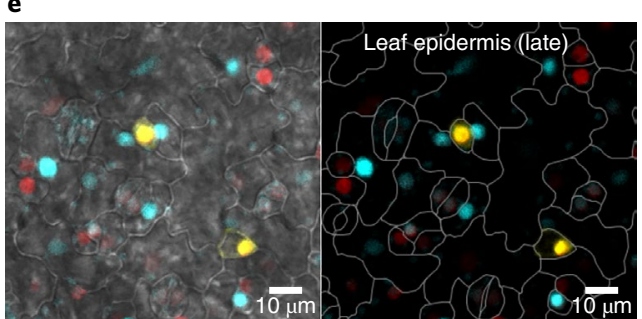

$\mathbf{g}$
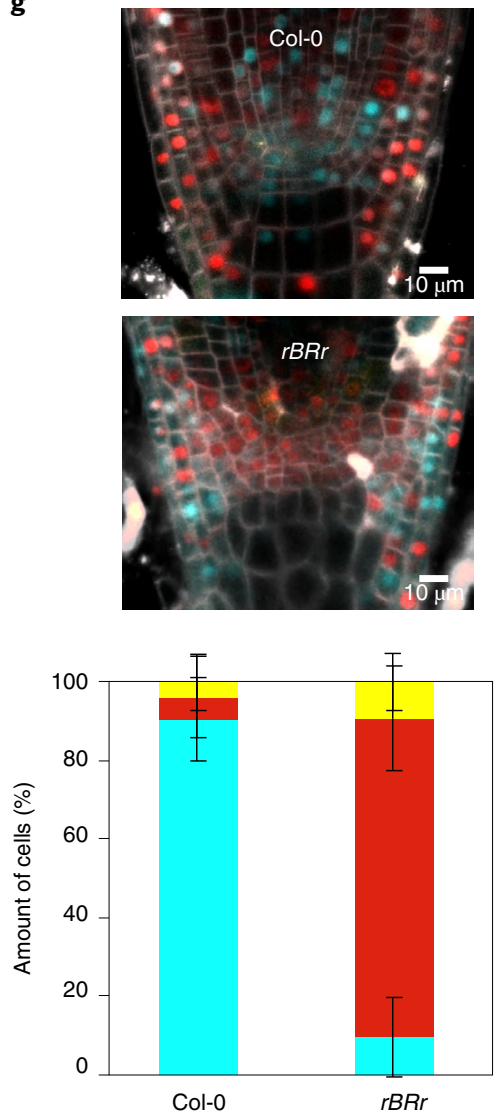

provide a useful combination of patterns to identify cells in G1, $\mathrm{S}+$ early G2, late G2 + prophase + metaphase and in later mitotic stages, according to the colour key shown in Fig. 1d. The labelling pattern of CDT1a-CFP and H3.1-mCherry allowed the identification of domains in the root apical meristem containing cells with high proliferation potential (G1 with H3.1-mCherry; Fig. 1e,f) and cells undergoing their last cell cycle before differentiation (G1 without H3.1-mCherry; Fig. 1e,g). A similar combination of patterns, 
Fig. 2 | Use of PlaCCl reporter in various experimental settings. a-c, Confocal images of a developing floral meristem (a), trichome (b) and petiole (c) of a leaf primordia. $\mathbf{d}, \mathbf{e}$, Confocal images of two different stages of leaf primordia development (early and late): cell cycle markers are expressed in all epidermal cells of recently emerged primordia (d) and are no longer expressed in differentiated cells at later stages (e). $\mathbf{f}$, Use of $\mathrm{PlaCCl}$ line to evaluate a pharmacological treatment. Seedlings were treated with the TOR inhibitor AZD8055 (1 $\mu \mathrm{M})$ during $24 \mathrm{~h}$ and imaged by confocal microscopy after staining the plasma membrane with FM4-64 (0.5 mM). LRC, lateral root cap. The amount of cells in different cell cycle phases was estimated by measuring the signal of individual channels (see Methods). Proliferating cells in the root apical meristem (RAM) epidermis of control ( $n=3$ roots, $n=369$ cells) and treated seedlings ( $n=4$ roots, $n=440$ cells) were scored (bottom panel). Error bars indicate the standard deviation. The $P$-values for the two-tailed unpaired $t$-test corrected for multiple comparisons using Holm-Sidak method were 0.0007, 0.0105 and 0.1380 for G1, S+early G2 and late G2 + M phases, respectively, at $95 \%$ confidence. $\mathbf{g}$, Use of $\mathrm{PlaCCl}$ line in a mutant background. Seedlings of wild type (Col-0) and $r B R r$ mutant expressing an interference RNA ( $\mathrm{RNAi}$ ) targeted against RBR1 in the root meristem with the $R C H 1$ promoter $(R C H 1:: R B R \mathrm{RNAi}$ ) and the PlaCCl markers were grown for $7 \mathrm{~d}$ and cells in different cell cycle phases were analysed. The amount of cells in the distal part of the RAM containing stem cells and early derivatives, was scored (Col-0, $n=11$ roots, $n=91$ cells; $r B R r, n=16$ roots, $n=133$ cells). Error bars indicate the standard deviation. The $P$-values for the two-tailed unpaired $t$-test corrected for multiple comparisons using Holm-Sidak method were $<0.000001,<0.000001$ and 0.073 for G1, S+early G2 and late G2 + M, respectively, at $95 \%$ confidence. a-g, Experiments were repeated at least three times with similar results.

without the CYCB1;1-YFP marker allowed the identification of cells in G- and S-phase during the endocycle (Fig. 1e,h).

To confirm these observations, we analysed how the PlaCCI reporters correlate with EdU that labelled cells in S-phase, an approach done previously for other cell cycle studies ${ }^{9,10}$. We confirmed that CDT1a-CFP and EdU signals anticorrelate, supporting the robustness of this reporter to identify G1 cells (Fig. 1i, upper panel, and Supplementary Fig. 4). Both early/mid and late S-phase nuclei contained H3.1-mCherry, which is maintained in G2 (Fig. 1i, upper panel). Quantification of individual signals revealed that EdU-labelled nuclei, classified as early/mid and late S-phase depending on the EdU pattern, contained a range of H3.1-mCherry signals (Fig. 1i, lower panel), as expected from H3.1 being incorporated and accumulated during S-phase. A similar pattern was observed for EdU-negative nuclei (Fig. 1i, lower panel). However, CDT1a-containing nuclei possessed a smaller amount of H3-1-mCherry.

Several results strongly ruled out that the expression of the reporter genes might be deleterious for plant growth. First, the rosette areas of both wild-type Col-0 and PlaCCI lines were indistinguishable (Supplementary Fig. 5a) and kinetic experiments showed that root growth was unaffected in the PlaCCI line (Supplementary Fig. 5b). At the cellular level, we measured root meristem size and found that there were no substantial differences between PlaCCI and wild-type roots (Fig. 1j). Although constitutive overexpression of CDT1a increases the ploidy level of endoreplicated cells ${ }^{12}$, CDT1a expression from its own promoter either in the wild type ${ }^{11}$ or in the PlaCCI line did not produce detectable changes in the ploidy profile in 28-day-old leaves (Fig. 1k and Supplementary Fig. 6). We also measured cell size in the adaxial leaf epidermis of 28-day-old leaves (leaf no. 3/4) and found that the cell size distributions of Col-0 and PlaCCI plants were not statistically different (Fig. 11). Thus, we conclude that expression of reporter proteins in the PlaCCI line does not produce major phenotypic changes.

The cell cycle markers are expressed not only in the root meristem but in all organs analysed; for example, shoot apical or floral meristems (Fig. 2a), developing trichomes (Fig. 2b) or the petiole of developing primordia (Fig. 2c). Also, the observation of two stages of leaf development clearly showed that cell cycle markers were expressed in almost every epidermal cell at early stages of leaf development (Fig. 2d) but disappeared in differentiated cells at later stages (Fig. 2e).

In this context, we foresee that the PlaCCI line constitutes a handy tool for cell proliferation studies in different organs and can be useful to assess cell proliferation dynamics after a variety of treatments. To confirm this, we carried out two different experiments. One, a pharmacological treatment of PlaCCI plants with the TOR (TARGET OF RAPAMYCIN) kinase inhibitor AZD8055 that affects root growth ${ }^{16}$. We observed an increase in the amount of cells labelled with CDT1a-CFP, effectively visualizing a significant G1 delay (Fig. 2f), consistent with the G1 arrest observed after TOR inhibition in yeast ${ }^{17}$. Another, by expressing the PlaCCI markers in a $r B R r$ mutant background, with a defect in the retinoblastoma-related (RBR1) protein, a general repressor of G1 progression ${ }^{18-20}$, which increases the division rate of stem cells ${ }^{21}$. The high number of cells marked with CDT1a-CFP in the wild type is significantly reduced in the $r B R r$ mutant as a result of cells moving faster through G1 (Fig. $2 \mathrm{~g}$ ).

We conceived the PlaCCI line in its simple form to facilitate its widespread use. However, it can eventually be used in combination with procedures to visualize cell walls or membranes (with fluorescent staining methods or by crossing with appropriate markers). Although this is possible, the need to distinguish four (or more) fluorescent markers is a limitation for certain confocal settings. Additionally, the lag in reaching detectable amounts of CDT1a-CFP after the end of mitosis means that very early G1 cells could not be directly identified. This could be solved indirectly by evaluating the size and shape of cells with no CDT1a-CFP (with or without H3.1-mCherry) that, if in early G1, should appear as pairs of small cells as a result of mitosis. The use of live-imaging can be very helpful in these cases. Finally, a new generation of PlaCCI lines should ideally contain a marker expressed at the G1/S transition and degraded upon S-phase termination that would allow unequivocal identification of 'only' S-phase cells. However, this is not available yet. In any case, we believe that the current PlaCCI reporter will become a tool of choice to determine the influence of cell cycle dynamics in developing processes. Furthermore, we anticipate that the strategy used here could be adapted to other plant species.

\section{Methods}

Cloning procedures and generation of transgenic plants. The Goldenbraid (GB) system GB3.0 was used for the generation of the expression cassette in the transformation plasmid ${ }^{15}$. The different modules containing promoters and genes were PCR amplified on the basis of published information ${ }^{7,11,13}$. Primers were designed using the GB-domesticating tool available online (gbcloning.upv.es) and are listed in Supplementary Table 1. NPTII transcriptional unit, YFP and 35S, HSP18.2 terminators were obtained from GB (GB0226, GB1182, GB0036 and GB0035). Each domesticated fragment was cloned into the pUPD or pUPD2 vector, and the transcriptional units and multipartite constructs were subsequently assembled as described in Supplementary Fig. 2 and Fig. 1a. All constructs were confirmed by sequencing (Macrogen). The A. tumefasciens $\mathrm{C} 58 \mathrm{C} 1$ strain was transformed with the final multipartite plasmid (pDGB3_ $\Omega 1$ ) containing the four transcription units and used to transform Arabidopsis Col-0 plants by the floral dip $\operatorname{method}^{22}$. Transgenic plants were selected with kanamycin.

Live imaging and confocal microscopy. Sterilized seeds were stratified during $2 \mathrm{~d}$ at $4^{\circ} \mathrm{C}$ and sown on plates containing $0.5 \times \mathrm{MS}$ medium ( $\mathrm{pH} 5.7$ ) supplemented with MES, vitamins, $1 \%$ sucrose and $1 \%$ plant agar (Duchefa). Plants were grown at $21^{\circ} \mathrm{C}$ and $60 \%$ moisture, under long day conditions ( $16 \mathrm{~h}$ light, $8 \mathrm{~h}$ dark). Four-day-old plants were transferred to a P35 glass bottom dish, covered with a piece of $1 \%$ agar MSS and acclimated vertically for $4-10 \mathrm{~h}$ in the growth chamber. Stack images of the root meristem ( $3-\mu \mathrm{m}$ section) were acquired every $30 \mathrm{~min}$ up to $8 \mathrm{~h}$ using a $\times 40$ oil objective with a NIKON A1R + confocal microscope. Tile-scanning $(2 \times 1$ tiles $)$ was used to ensure the imaging of the whole meristem. Images and video editing were performed using FIJI (ref. ${ }^{23}$ ). For treatment with TOR inhibitor, plants were grown during $5 \mathrm{~d}$ in MSS medium and transferred for $24 \mathrm{~h}$ in a medium containing $1 \mu \mathrm{M}$ of AZD8055 (Sigma) and imaged as described above. 
EdU-labelling and immunofluorescence assay. Five-day-old seedlings were incubated in liquid MSS containing $20 \mu \mathrm{M}$ EdU for $15 \mathrm{~min}$. Plants were fixed with $4 \%$ paraformaldehyde and immunodetection was performed as described previously ${ }^{13}$. Antibodies raised against GFP, PGAB1 (Chromotek, 1/2,000) were used to detect CDT1a-eCFP and did not recognize CYCB1;1-YFP in our settings. HTR13-mCherry was detected with a rat anti-RFP antibody (clone 5F8, 1/1000, Chromotek). Anti-rabbit AlexaFluor-488 and anti-rat AlexaFluor-555 (ThermoScientific, 1/1,000) were used as secondary antibodies. EdU incorporation was detected using the Click-iT Plus EdU AlexaFluor 647 Imaging kit (Invitrogen) following manufacturer instructions.

Quantification of fluorescent signals defining cell cycle phases. Cell cycle phases were primarily defined on the basis of the fluorescent signal of each marker protein scored for individual cells in a time lapse imaging experiment using FIJI (ref. ${ }^{23}$ ). The values for mean intensity signals were then processed according to the colour key shown in Fig. 1d. It is advisable to confirm visually that definition of each cell correspond to the image, since automatic measurement after segmentation is not a safe procedure due the eventual spread of fluorescent signal from neighbour cells.

Flow cytometry. Leaves no. 3/4 of 28-day-old plants grown in soil were chopped with a razor blade in $500 \mu \mathrm{l}$ of nuclear isolation buffer ${ }^{19}$. RNAse at $100 \mu \mathrm{g} \mathrm{ml}^{-1}$ and propidium iodide at $50 \mu \mathrm{g} \mathrm{ml}^{-1}$ were added and nuclei were analysed using a FACSCanto A flow cytometer (Becton Dickinson). Analyses were performed with FloJo software.

Measurement of root meristem length. Roots of 5-day-old plants were quickly stained in a lugol solution and mounted in a chloral hydrate/glycerol/water medium $(80 / 10 / 10, \mathrm{p} / \mathrm{v} / \mathrm{v})$. Roots were imaged in the central plane with an Axioskop2 microscope (Zeiss) coupled with a DMC6200 camera (Leica) using a dry $\times 20$ Nomarsky objective. Measurements were performed using FIJI (ref. ${ }^{23}$ ).

Measurement of cell size in the leaf epidermis. Leaves no. 3/4 of 28-day-old plants were submerged in a $90 \%$ lactic acid solution and incubated at $95^{\circ} \mathrm{C}$ for $15 \mathrm{~min}$. Samples were left $24 \mathrm{~h}$ at room temperature and then mounted in $90 \%$ lactic acid for microscopic observation. Leaf epidermis was imaged with an Axioskop2 microscope (Zeiss), as described for root meristem length measurements.

Reporting Summary. Further information on research design is available in the Nature Research Reporting Summary linked to this article.

\section{Data availability}

Source data are provided with this paper.

Received: 31 January 2020; Accepted: 14 August 2020;

Published online: 28 September 2020

\section{References}

1. Sakaue-Sawano, A. et al. Visualizing spatiotemporal dynamics of multicellular cell-cycle progression. Cell 132, 487-498 (2007).

2. Sugiyama, M. et al. Illuminating cell-cycle progression in the developing zebrafish embryo. Proc. Natl Acad. Sci. USA 106, 20812-20817 (2009).

3. Zielke, N. et al. Fly-FUCCI: a versatile tool for studying cell proliferation in complex tissues. Cell Rep. 7, 588-598 (2014).

4. Bajar, B. T. et al. Fluorescent indicators for simultaneous reporting of all four cell cycle phases. Nat. Methods 13, 993-996 (2016).

5. Caro, E., Castellano, M. M. \& Gutierrez, C. A chromatin link that couples cell division to root epidermis patterning in Arabidopsis. Nature 447, 213-217 (2007).

6. Caro, E. \& Gutierrez, C. A green GEM: intriguing analogies with animal geminin. Trends Cell Biol. 17, 580-585 (2007).

7. Colon-Carmona, A., You, R., Haimovitch-Gal, T. \& Doerner, P. Technical advance: spatio-temporal analysis of mitotic activity with a labile cyclin-GUS fusion protein. Plant J. 20, 503-508 (1999).

8. Adachi, S. et al. Programmed induction of endoreduplication by DNA double-strand breaks in Arabidopsis. Proc. Natl Acad. Sci. USA 108, 10004-10009 (2011).
9. Yin, K. et al. A dual-color marker system for in vivo visualization of cell cycle progression in Arabidopsis. Plant J. 80, 541-552 (2014).

10. Jones, A. R. et al. Cell-size dependent progression of the cell cycle creates homeostasis and flexibility of plant cell size. Nat. Commun. 8, 15060 (2017).

11. Desvoyes, B. et al. FBL17 targets CDT1a for degradation in early S-phase to prevent Arabidopsis genome instability. Preprint at https://www.biorxiv.org/ content/10.1101/774109v1 (2019).

12. Castellano, M. M., Boniotti, M. B., Caro, E., Schnittger, A. \& Gutierrez, C. DNA replication licensing affects cell proliferation or endoreplication in a cell type-specific manner. Plant Cell 16, 2380-2393 (2004).

13. Otero, S., Desvoyes, B., Peiro, R. \& Gutierrez, C. Histone H3 dynamics uncovers domains with distinct proliferation potential in the Arabidopsis root. Plant Cell 28, 1361-1371 (2016).

14. Ubeda-Tomas, S. et al. Gibberellin signaling in the endodermis controls Arabidopsis root meristem size. Curr. Biol. 19, 1194-1199 (2009).

15. Sarrion-Perdigones, A. et al. GoldenBraid: an iterative cloning system for standardized assembly of reusable genetic modules. PLoS ONE 6, e21622 (2011).

16. Montané, M.-H. \& Menand, B. ATP-competitive mTOR kinase inhibitors delay plant growth by triggering early differentiation of meristematic cells but no developmental patterning change. J. Exp. Bot. 64, 4361-4374 (2013).

17. Barbet, N. C. et al. TOR controls translation initiation and early G1 progression in yeast. Mol. Biol. Cell 7, 25-42 (1996).

18. Park, J. A. et al. Retinoblastoma protein regulates cell proliferation, differentiation, and endoreduplication in plants. Plant J. 42, 153-163 (2005).

19. Desvoyes, B., Ramirez-Parra, E., Xie, Q., Chua, N. H. \& Gutierrez, C. Cell type-specific role of the retinoblastoma/E2F pathway during Arabidopsis leaf development. Plant Physiol. 140, 67-80 (2006).

20. Harashima, H. \& Sugimoto, K. Integration of developmental and environmental signals into cell proliferation and differentiation through RETINOBLASTOMA-RELATED 1. Curr. Opin. Plant Biol. 29, 95-103 (2016).

21. Wildwater, M. et al. The RETINOBLASTOMA-RELATED gene regulates stem cell maintenance in Arabidopsis roots. Cell 123, 1337-1349 (2005).

22. Clough, S. J. \& Bent, A. F. Floral dip: a simplified method for Agrobacterium-mediated transformation of Arabidopsis thaliana. Plant J. 16 735-743 (1998)

23. Rueden, C. T. et al. ImageJ2: ImageJ for the next generation of scientific image data. BMC Bioinforma. 18, 529 (2017).

\section{Acknowledgements}

We thank the Confocal Microscopy and the Cytometry services of CBMSO for support, E. Martinez-Salas for comments, S. Herrero-Desvoyes for help with cell size measurements, E. Caro for the terminator modules for GoldenBraid constructs, V. Mora-Gil for technical assistance and members of the laboratory for continuous feedback. Research in our laboratory is supported by grant nos. BIO2017-92329-EXP and RTI2018-094793-B-I00 from MICIU, and ERC-2018-AdG_833617 from European Union, and by institutional grants from Banco de Santander and Fundación Ramon Areces to the Centro de Biología Molecular Severo Ochoa.

\section{Author contributions}

B.D. and C.G designed this work. A.A.-E. and M.D.B. participated in the initial cloning steps. B.D. performed all the experiments. B.D. and C.G. wrote the manuscript.

\section{Competing interests}

The authors declare no competing interests.

\section{Additional information}

Supplementary information is available for this paper at https://doi.org/10.1038/ s41477-020-00770-4.

Correspondence and requests for materials should be addressed to B.D. or C.G. Peer review information Nature Plants thanks Lieven De Veylder and the other, anonymous, reviewer(s) for their contribution to the peer review of this work.

Reprints and permissions information is available at www.nature.com/reprints.

Publisher's note Springer Nature remains neutral with regard to jurisdictional claims in published maps and institutional affiliations.

(c) The Author(s), under exclusive licence to Springer Nature Limited 2020 


\section{natureresearch}

\section{Reporting Summary}

Nature Research wishes to improve the reproducibility of the work that we publish. This form provides structure for consistency and transparency in reporting. For further information on Nature Research policies, see Authors \& Referees and the Editorial Policy Checklist.

\section{Statistics}

For all statistical analyses, confirm that the following items are present in the figure legend, table legend, main text, or Methods section.

$\mathrm{n} / \mathrm{a} \mid$ Confirmed

\ The exact sample size $(n)$ for each experimental group/condition, given as a discrete number and unit of measurement

$\square \bigotimes$ A statement on whether measurements were taken from distinct samples or whether the same sample was measured repeatedly

$\square$ The statistical test(s) used AND whether they are one- or two-sided

Only common tests should be described solely by name; describe more complex techniques in the Methods section.

Х $\square$ A description of all covariates tested

Х $\square$ A description of any assumptions or corrections, such as tests of normality and adjustment for multiple comparisons

$\triangle$ A full description of the statistical parameters including central tendency (e.g. means) or other basic estimates (e.g. regression coefficient)

AND variation (e.g. standard deviation) or associated estimates of uncertainty (e.g. confidence intervals)

For null hypothesis testing, the test statistic (e.g. $F, t, r$ ) with confidence intervals, effect sizes, degrees of freedom and $P$ value noted

Х Give $P$ values as exact values whenever suitable.

\ $\square$ For Bayesian analysis, information on the choice of priors and Markov chain Monte Carlo settings

Х $\square$ For hierarchical and complex designs, identification of the appropriate level for tests and full reporting of outcomes

Х $\square$ Estimates of effect sizes (e.g. Cohen's $d$, Pearson's $r$ ), indicating how they were calculated

Our web collection on statistics for biologists contains articles on many of the points above.

\section{Software and code}

Policy information about availability of computer code

\section{Data collection Cytometry: BD FACS DIVA v8.0.1}

Confocal Microscopy: NIkon Nis element 5.20.01

Bright field microscopy: Camera Leica LAS v4.13

Data analysis

\section{Flow cytometry: FlowJo v10}

Image analysis: Fiji ImageJ 2.0.0-rc69/1.52i

Data analysis: Prism (version 8.2.1) and Excel (version 16.16.12)

For manuscripts utilizing custom algorithms or software that are central to the research but not yet described in published literature, software must be made available to editors/reviewers. We strongly encourage code deposition in a community repository (e.g. GitHub). See the Nature Research guidelines for submitting code \& software for further information.

\section{Data}

Policy information about availability of data

All manuscripts must include a data availability statement. This statement should provide the following information, where applicable:

- Accession codes, unique identifiers, or web links for publicly available datasets

- A list of figures that have associated raw data

- A description of any restrictions on data availability

A data availability statement has been included, as well as an Excel file with raw data used for calculations for each relevant figure. 


\section{Field-specific reporting}

Please select the one below that is the best fit for your research. If you are not sure, read the appropriate sections before making your selection. $\bigotimes$ Life sciences $\quad \square$ Behavioural \& social sciences $\quad \square$ Ecological, evolutionary \& environmental sciences

For a reference copy of the document with all sections, see nature.com/documents/nr-reporting-summary-flat.pdf

\section{Life sciences study design}

All studies must disclose on these points even when the disclosure is negative.

Sample size No statistical method was used to predetermine the sample size. For each type of experiment or repeat, different sample sizes (normally between 5 and 10) were analyzed, based on our previous experience (Otero et al, Plant Cell 2016; Desvoyes et al., BioRxiv 2019)

Video analysis (of $\geq 8 \mathrm{~h}$ long) were carried out using $>10$ independent experiments. Videos of $<8 \mathrm{~h}$ long were $>10$ independent experiments. In addition, a large number of individual images were analyzed.

Data exclusions No data were excluded from the analysis

Replication All attempts at replicating experiments were successful. In each case, one of them was presented in the figures.

Randomization Transgenic plants were randomly chosen in the selection plates after transforamtion and confirmed to have similar levels of fluorescence signals for all the markers used. Five independent Arabidopsis PlaCCI lines were analyzed.

Blinding Blinding was not used in this study. To avoid any bias, we have quantified all samples available in each experiment (e.g., several root meristems, as indicated in each figure).

\section{Reporting for specific materials, systems and methods}

We require information from authors about some types of materials, experimental systems and methods used in many studies. Here, indicate whether each material, system or method listed is relevant to your study. If you are not sure if a list item applies to your research, read the appropriate section before selecting a response.

Materials \& experimental systems

\begin{tabular}{|c|c|}
\hline$n / a$ & Involved in the study \\
\hline & $\bigotimes$ Antibodies \\
\hline Х & Eukaryotic cell lines \\
\hline Х & Palaeontology \\
\hline Х & Animals and other organisms \\
\hline Х & Human research participants \\
\hline 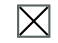 & Clinical data \\
\hline
\end{tabular}

Methods $\mathrm{n} / \mathrm{a}$ Involved in the study

$\bigotimes \square$ ChIP-seq

\Flow cytometry

$\bigotimes \square$ MRI-based neuroimaging

\section{Antibodies}

Antibodies used

Anti-GFP, pabg1-100, Lot 70828032AB, Chromotek

Anti-RFP, 5F8, Lot 60706002AB, Chromotek

Alexa Donkey anti-Rabbit 488, Lot 1981155

Alexa Goat anti-Rat 555, Lot 1987272

Validation

Anti-GFP and anti-RFP did not give any signal in wild type Arabidopsis plants, not expressing GFP or RFP. Alexa samples are regularly tested in our Antibody service 


\section{Plots}

Confirm that:

$\bigotimes$ The axis labels state the marker and fluorochrome used (e.g. CD4-FITC).

$\bigotimes$ The axis scales are clearly visible. Include numbers along axes only for bottom left plot of group (a 'group' is an analysis of identical markers).

$\bigotimes$ All plots are contour plots with outliers or pseudocolor plots.

$\bigotimes$ A numerical value for number of cells or percentage (with statistics) is provided.

\section{Methodology}

Sample preparation

It is described in Methods. Leaves \#3/4 of 28 day-old plants grown in soil were chopped with a razor blade in $500 \mu$ l of nuclear isolation buffer, as described (Desvoyes et al., Plant Physiol, 2006). RNAse at $100 \mu \mathrm{g} / \mathrm{mL}$ and propidium iodide at $50 \mu \mathrm{g} / \mathrm{mL}$ was added before being analyzed in the flow cytometer

Instrument

FACSCanto II (Becton Dickinson)

Software

BD FACS DIVA v8.0.1

FlowJo

Cell population abundance

These numbers are provided in Supplementary Fig. 6, where the gating strategy is detailed. In all cases a minimum of 10,000 nuclei were analyzed.

Gating strategy

It is described in Supplementary Fig. 6 (panels a-f). Two gating criteria were used: propidium iodide (FL2-A)/side scatter area (SSC-A) in a log scale to analyze the ploidy of nuclei $(a, d)$ and propidium iodide FL3-A/FL3-W in a linear scale to discriminate and exclude nuclei doublets $(b, e)$. Representative ploidy profiles are presented $(c, f)$. Percentage of gated events $(a, d)$ and/or number of analyzed nuclei $(b, e)$ are presented in each panel.

$\bigotimes$ Tick this box to confirm that a figure exemplifying the gating strategy is provided in the Supplementary Information. 